\title{
Effect of respiratory suspension on the computation of volume-based early peak filling rate to late peak filling rate ratio
}

\author{
Amol Pednekar ${ }^{1 *}$, Jiming Zhang ${ }^{2}$, Claudio Arena ${ }^{2}$, Melissa Andrews², Debra Dees², Benjamin Cheong ${ }^{2}$, \\ Raja Muthupillai ${ }^{2}$
}

From 19th Annual SCMR Scientific Sessions

Los Angeles, CA, USA. 27-30 January 2016

\section{Background}

In the intact circulation, changes in intrathoracic pressure and/or lung volume will simultaneously induce alterations in cardiac volumes, output, and contractility among other alterations [1]. In this study, we evaluate the impact of respiratory suspension on the computation of volume-based early peak filling rate (EPFR) to late peak filling rate (LPFR) ratio using peak velocitybased Doppler echo measured early peak velocity (E) to peak velocity during atrial contraction (A) measured at the tip of the mitral leaflets as the reference.

\section{Methods}

All imaging for this IRB approved prospective study was performed on a $1.5 \mathrm{~T}$ commercial MR scanner (Achieva, Philips Healthcare) in 27 volunteers $(16 \mathrm{~m} / 16 \mathrm{f}$; age 48 (20-66)yrs). MRI: Identical imaging parameters were used for breath held (BH) (17 subjs), and free breathing (FB) (10 subjs) cine SSFP sequences (TR/TE/flip angle: $\left.3 / 1.5 / 60^{\circ}\right)$; acqd voxel size: $2.25 \times 2.25 \times 8 \mathrm{~mm}^{3}$; SENSE:2, temp res: $10-15 \mathrm{~ms}$; acq time: $18 \mathrm{RR}$ intervals/ slice; covering the LV in short-axis orientation. FB pulse sequence is described in [2]. Echocardiography: Subjects were transported to ultrasound (Philips Healthcare, IE 33) on the same scanner bed to minimize physiologic variation and E/A ratio was obtained. Data Analysis: CMR expert drawn endocardial contour at end diastole was propagated across the cardiac phases by a semiautomated algorithm. Resultant LV contours were manually adjusted by CMR expert if needed. From these contours time-LV volume curve was further analyzed

Philips Healthcare, Houston, TX, USA

Full list of author information is available at the end of the article using custom-written software in MATLAB ${ }^{\mathrm{TM}}$. The raw LV volume curve was upsampled by a factor of 4 , and the derivative of the time-volume curve was estimated using the method described in [3]. We defined the ratio of EPFR to LPFR as the MR equivalent surrogate of velocity based echo index of $E$ over A ratio. Linear regression and Bland-Altman (BA) analysis was performed on the results obtained with MR and echo to obtain slope $(\mathrm{m})$, coefficient of determination $\left(\mathrm{r}^{2}\right)$, bias (mean of difference), and limits of agreement(LA, 1.96* stdev of diff).

\section{Results}

High frame rate cine SSFP sequence during free breathing provides cine MR images with adequate temporal resolution to estimate MR based index (EPFR/LPFR) of diastolic function. Doppler based E/A ratios were in good agreement with EPFR/LPFR for FB $\left(\mathrm{m}=1.07, \mathrm{r}^{2}=\right.$ 0.85 , bias $=0.18$, LA 0.12). Breath held acquisitions correlated well with Doppler based E/A ratio $\left(\mathrm{m}=1.82, \mathrm{r}^{2}=\right.$ 0.69 , bias $=0.22$ ) however LA was more than 8 times higher than with FB acquisition. The BA analysis showed a slope of 0.66 for the bias.

\section{Conclusions}

The volume based E/A ratio derived from high temporal resolution cine MR correlated well with velocity based E/A ratio from echo. The complex interactions between respiratory and cardiovascular systems have direct impact on the measurement of volume-based EPFR/ LPFR. EPFR/LPFR computed using free breathing acquisitions are in very good agreement with E/A from echo. 


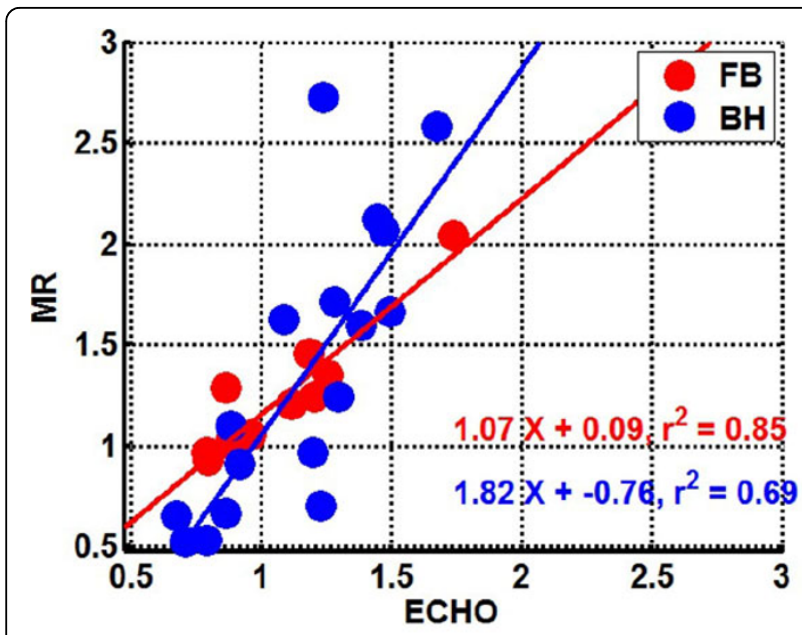

(A)

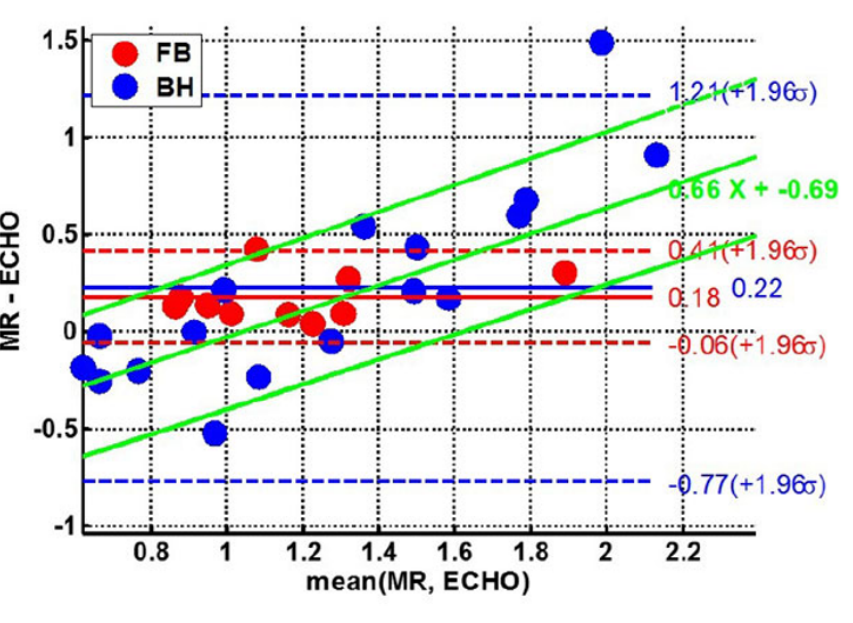

(B)

Figure 1 Linear regression (A) and Bland-Altman (B) for echo (E/A) verses MR (EPFR/LPFR) ratios using breath held (Blue) and free breathing (Red) high temporal resolution cine SFFP sequences.

\section{Authors' details}

${ }^{1}$ Philips Healthcare, Houston, TX, USA. ${ }^{2}$ Radiology, CHI St. Luke's Health,

Houston, TX, USA.

Published: 27 January 2016

\section{References}

1. Lung: 1981, 159:175-186.

2. ISMRM 2012, 3938.

3. JMRI 2010, 31(4):872-880.

\section{doi:10.1186/1532-429X-18-S1-093}

Cite this article as: Pednekar et al:: Effect of respiratory suspension on the computation of volume-based early peak filling rate to late peak filling rate ratio. Journal of Cardiovascular Magnetic Resonance 201618 (Suppl 1):093.

Submit your next manuscript to BioMed Central and take full advantage of:

- Convenient online submission

- Thorough peer review

- No space constraints or color figure charges

- Immediate publication on acceptance

- Inclusion in PubMed, CAS, Scopus and Google Scholar

- Research which is freely available for redistribution 